\title{
La individualización del aprendizaje y el trabajo cooperativo mediante las Tecnologías de la Información y la Comunicación en Educación (TICE). Desarrollo de las competencias profesionales en los estudiantes de $2^{\circ}$ curso del Grado de Maestros en Educación Primaria
}

\author{
Álvaro MARTín ESPINOSA \\ Universidad de Castilla-La Mancha \\ alvaro.martin@uclm.es
}

\begin{abstract}
Resumen
El Espacio Europeo de Educación Superior (EEES) conlleva una reflexión acerca de la metodología docente universitaria. Los estudiantes deben adquirir unas competencias profesionales que parece difícil puedan conseguir con la práctica docente tradicional, exigiendo un cambio de roles tanto en docentes como en los futuros titulados, mucho más preocupados por aprobar que por realmente aprender. El docente debe ser un mediador entre el discente y su proceso de enseñanza-aprendizaje exigiendo irremediablemente un mayor grado de protagonismo y autonomía del alumnado, favoreciendo así su aprendizaje.
\end{abstract}

Palabras clave: EEES, competencias profesionales, individualización, portafolio digital.

Individualized learning and cooperative work through Information and Communication Technologies in Education (ICTE). Development of professional competences in the $2^{\text {nd }}$ course of the primary school teachers degree.

\begin{abstract}
The European Higher Education Area (EHAE) entails, among other things, a reflection on the university teaching methodology. The students must acquire some professional competences, and they are not very likely to acquire them with the traditional teaching methods. Both professors and students need to play new roles. At present, the majority of these students are more interested in passing than in learning. The professor should be a mediator between the students and their teaching-learning process. In which the students must have more prominence and autonomy. That will benefit their learning.
\end{abstract}

Keywords: EHEA, professional competences, individualized programmes, digital portfolio.

\section{Referencia normalizada:}

Martín Espinosa: A. (2013) La individualización del aprendizaje y el trabajo cooperativo mediante las Tecnologías de la Información y la Comunicación en Educación (TICE). Desarrollo de las competencias profesionales en los estudiantes de $2^{\circ}$ curso del Grado de Maestros en Educación Primaria. Historia y Comunicación Social. Vol. 18. Nº Especial Diciembre. Págs. 765-781. 
Sumario: 1. Introducción. 2. Metodología de studio. 3. Planificación y puesta en marcha. 4. Resultados. 5. Conclusiones. 6. Referencias bibliográficas. 7. Notas.

\section{Introducción}

El Espacio Europeo de Educación Superior (EEES) conlleva, entre otras muchas cosas, una reflexión acerca de la metodología docente universitaria. Los estudiantes deben adquirir unas competencias profesionales que parece difícil puedan adquirir con la práctica docente tradicional, exigiendo un cambio de roles tanto en docentes como en los futuros titulados, mucho más preocupados por aprobar que por realmente aprender. Por un lado, el docente debe ser un mediador entre el discente y su proceso de enseñanza-aprendizaje exigiendo irremediablemente un mayor grado de protagonismo, autonomía e iniciativa personal del mismo. En este artículo presento una investigación puesta en marcha durante el curso académico 2012-2013 que tiene por objetivo valorar las aportaciones de la metodología de la individualización del aprendizaje y el trabajo cooperativo para lograr una mejora en la adquisición de competencias en dos grupos de alumnos de $2^{\circ}$ curso del Grado de Magisterio en Educación Primaria. Se han utilizado para su valoración diversas escalas de registro y observación, guías explicativas, tutorías individualizadas y grupales así como un cuestionario elaborado para tal fin, donde los alumnos han valorado la metodología empleada en las dos asignaturas, la importancia de la motivación en el proceso de aprendizaje individualizado, el interés por las tareas propuestas así como el grado de satisfacción con el conocimiento adquirido. La herramienta principal de trabajo autónomo ha sido el portafolios digital y la tarea grupal cooperativa ha sido un trabajo de investigación acerca de un tema relacionado con las asignaturas pero que los propios estudiantes elegían libremente en función de sus intereses y motivaciones, exponiendo las conclusiones de los mismos al resto de compañeros y al profesor. Se recogen en este artículo los resultados más significativos pretendiendo aportar una evidencia más a los ya buenos resultados obtenidos en otros estudios de similares características.

Teniendo presentes diversas investigaciones acerca de metodologías diferentes a la considerada tradicional donde el alumnado es un mero receptor de conocimientos y siendo consciente de que esa información transmitida oralmente es difícilmente aplicable correctamente a contextos reales y fácilmente olvidable a los pocos meses de superar la asignatura, en el presente artículo se pretende analizar de qué forma el alumnado motivado con propuestas de tareas individuales diferentes a las habituales y con un trabajo individual y colectivo mediado a través de pautas de trabajo autónomo y tutorías en pequeño grupo, podemos conseguir una mayor satisfacción del discente con el conocimiento adquirido a lo largo de la asignatura, su implicación en su proceso de enseñanza-aprendizaje así como aprender a cooperar en busca del bien común: conseguir una construcción social del conocimiento con las aportaciones de todos y cada uno de los miembros que forman parte de una comunidad de aprendizaje incluido, por su puesto, al docente que guía, orienta y ayuda a sus alumnos y alumnas 
en esta metodología todavía novedosa forma de aprender en la enseñanza universitaria. Quizá la competencia más importante desde este punto de vista sea la competencia de aprender a aprender, imprescindible para actualizar de forma autónoma las capacidades que cualquier docente, independientemente de la etapa educativa a la que se enfrente en su desempeño profesional y que deberá fomentar y potenciar en su alumnado a lo largo de su vida laboral.

Diversos son los estudios que avalan la utilización del portafolios como herramienta de aprendizaje individualizado y autónomo así como con muchas posibilidades para compartir conocimientos. En esta investigación se decidió introducir la utilización del portafolios digital (blogs) por el efecto innovador y motivador que podía representar para el alumnado. Investigaciones anteriores demuestran los buenos resultados obtenidos con esta estrategia metodológica en la mejora de los procesos de enseñanza-aprendizaje.

De esta forma, (Mokharti y Yellin, 1996) obtuvieron los siguientes resultados estudiando el uso del portafolio en la formación de docentes: 86\% de los estudiantes afirmaba que el portafolio promovía un aprendizaje más colaborativo, el $71 \%$ afirmaba que el portafolio incrementaba la actitud reflexiva del estudiante y el 63\% consideraba que ayudaba a establecer en clase un clima de seguridad.

(Dollase, 1996) resalta de igual forma que el uso de portafolios capacita al futuro profesor para mejorar habilidades tales como la propia organización de la tarea y la monitorización de la misma. En este sentido y teniendo presente la mejora de las competencias profesionales como objetivo fundamental, (Klenowski, 2000) concluyó en su estudio que el uso del portafolio en la formación de profesores: mejoraba el desarrollo de habilidades para la exposición pública y la actividad docente, las habilidades de autoevaluación e incrementaba las capacidades para el aprendizaje autónomo.

\section{Metodología del estudio}

El presente estudio es observacional descriptivo basado en los resultados de una encuesta contestada de forma anónima por 85 estudiantes que participaron en el desarrollo normalizado de dos asignaturas ( $96 \%$ del total) y que fue realizada tras la finalización del curso 2012-2013. Cabe señalar en este punto que 85 estudiantes repartidos en dos asignaturas ofrecen una ratio profesor alumno considerada baja en la actualidad, probablemente una de las causas más importantes para haber podido desarrollar este estudio de forma satisfactoria y que ha permitido el seguimiento individualizado de los portafolios digitales, la exposición de los trabajos grupales y la realización de tutorías en un número muy elevado en comparación con cursos pasados. Las asignaturas estudiadas son "Tendencias Contemporáneas de Educación” y "Educación y Sociedad", ambas cursadas en segundo curso del Grado de Maestro en Educación Primaria en la Facultad de Educación de Toledo pero que tienen unas características 
propias diferentes que resumiré a continuación. La primera es de contenidos más teóricos relacionados con la Historia de la Educación en España, pedagogos ilustres, tendencias educativas contemporáneas, etc. La segunda la podemos clasificar como más innovadora ya que se introducen contenidos relacionados con la educación actual dentro del marco de la sociedad de la información y la comunicación en la que todos estamos inmersos de una u otra manera. Es importante señalar que en ambas se utilizó la misma metodología y se pidieron las mismas tareas partiendo siempre del principio de individualización del aprendizaje y dando al alumnado un verdadero protagonismo, tanto en la elaboración de actividades individuales como en la realización de trabajos de forma cooperativa, con el objetivo de comprobar si los contenidos de las mismas podían condicionar de alguna forma los resultados de la investigación o si la realización de un portafolios digital podría encajar dentro de una asignatura como "Tendencias Contemporáneas de Educación". También resulta importante para la validación de los resultados obtenidos decir que la encuesta fue realizada anteriormente a la entrega de las calificaciones finales, intentando evitar de esta forma que el conocimiento de la calificación por parte de los estudiantes pudiera alterar o condicionar de algún modo sus respuestas. En ella, se valoraron quince ítems relacionados principalmente con el interés y la satisfacción del estudiante en diferentes aspectos, la opinión respecto a la puesta en práctica de la metodología de enseñanza-aprendizaje así como la adquisición de competencias profesionales útiles tanto en su formación como en su futuro profesional. La escala de respuesta contaba con cuatro valores: nada, poco, bastante y mucho. También se les pidió una valoración final numérica, en la que debían calificar la metodología empleada de forma global en una escala de 0 a 10 puntos.

Se introduce en este apartado la encuesta pasada al alumnado para la evaluación final de la metodología estudiada en ambas asignaturas (tabla 1). Como se puede comprobar fueron varios los ítem a valorar relacionados el interés y satisfacción con la metodología empleada, su percepción acerca de la mejora de algunas de las competencias importantes tanto en su formación como futuros docentes y su utilidad en el desempeño profesional, la valoración de las tareas propuestas inmersas en esta forma de trabajar y su calificación final de forma numérica con el objetivo principal de conocer las opiniones de los alumnos y alumnas respecto a esta metodología para poder, a parte de valorar sus satisfacción con el desarrollo de las asignaturas y el conocimiento adquirido al cursarlas, realizar una autoevaluación docente de cara a futuras mejoras o cambios que permitan perfeccionar y afianzar esta forma de trabajar dentro de las aulas universitarias. 
Tabla 1. Evaluación de la metodología utilizada en las asignaturas estudiadas

\begin{tabular}{|c|c|c|c|c|}
\hline ASPECTOS A VALORAR & Nada & Poco & Bast. & Mucho \\
\hline $\begin{array}{l}\text { 1. Interés por la actividad individual pro- } \\
\text { puesta }\end{array}$ & & & & \\
\hline $\begin{array}{l}\text { 2. Satisfacción con el conocimiento adqui- } \\
\text { rido }\end{array}$ & & & & \\
\hline $\begin{array}{l}\text { 3. Interés por la iniciación a la investigación } \\
\text { cualitativa por medio de entrevistas en pro- } \\
\text { fundidad. }\end{array}$ & & & & \\
\hline $\begin{array}{l}\text { 4. Interés por las actividades de trabajo } \\
\text { cooperativo propuestas. }\end{array}$ & & & & \\
\hline $\begin{array}{l}\text { 5. Satisfacción por elegir libremente los } \\
\text { temas a trabajar en equipo de forma coope- } \\
\text { rativa en función de los intereses o motiva- } \\
\text { ciones del grupo. }\end{array}$ & & & & \\
\hline $\begin{array}{l}\text { 6. Importancia de la tutorización o guía del } \\
\text { profesor. }\end{array}$ & & & & \\
\hline $\begin{array}{l}\text { 7. ¿Consideras importante partir de la } \\
\text { motivación individual para mejorar el } \\
\text { rendimiento académico? }\end{array}$ & & & & \\
\hline $\begin{array}{l}\text { 8. ¿Crees que esta metodología ha mejorado } \\
\text { tu competencia de autonomía e iniciativa } \\
\text { personal? }\end{array}$ & & & & \\
\hline $\begin{array}{l}\text { 9.¿Consideras que te será útil en tu futuro } \\
\text { profesional como docente? }\end{array}$ & & & & \\
\hline $\begin{array}{l}\text { 10. Coherencia entre la metodología docente } \\
\text { y la evaluación de la asignatura. }\end{array}$ & & & & \\
\hline $\begin{array}{l}\text { 11. ¿Consideras que esta metodología favo- } \\
\text { rece la competencia de aprender a aprender } \\
\text { y el aprendizaje a lo largo de la vida? }\end{array}$ & & & & \\
\hline $\begin{array}{l}\text { 12. ¿Consideras importante los debates y el } \\
\text { uso de foros para la construcción social del } \\
\text { conocimiento? }\end{array}$ & & & & \\
\hline $\begin{array}{l}\text { 13. ¿Crees que ayuda este tipo de activi- } \\
\text { dades a la búsqueda y discriminación de } \\
\text { la información por diversos medios (TIC, } \\
\text { libros, artículos...? }\end{array}$ & & & & \\
\hline $\begin{array}{l}\text { 14. Interés por conocer las normas APA para } \\
\text { la elaboración de trabajos evitando plagios. }\end{array}$ & & & & \\
\hline $\begin{array}{l}\text { 15. ¿Consideras necesaria una prueba obje- } \\
\text { tiva (examen oral o escrito) para la evalua- } \\
\text { ción final. }\end{array}$ & & & & \\
\hline \multicolumn{3}{|c|}{ 16. Valoración global de la metodología (Puntúa en una escala del 0 a 10) } & & \\
\hline SEXO: $\quad$ EDAD: & & & & \\
\hline
\end{tabular}

Elaboración propia. Cuestionario de evaluación de la metodología "individualización del aprendizaje y aprendizaje cooperativo". 


\section{Planificación y puesta en marcha}

Al ser una metodología no demasiado conocida por los estudiantes (algo curioso cuando ya deberían llegar de las etapas educativas inferiores acostumbrados a trabajar de esta forma), se decidió impartir al grupo completo varias sesiones mediante metodología más tradicional (clase magistral) dando a conocer la citada metodología de trabajo, la importancia para la adquisición de aprendizajes duraderos y los principales principios en los que se sustenta, como partir de la motivación e interés de cada alumno, la construcción personal y social del conocimiento con la mediación del profesor y la importancia de romper con esquemas mentales previos como la de sentarse a escuchar clases magistrales y tomar apuntes, tomando la palabra del profesor como algo inalterable y que deben reproducir en el examen así como la importancia de consultar y citar bibliografía acerca de los temas a tratar para enriquecer sus conocimientos. La finalidad de estas sesiones fue principalmente no desterrar de un plumazo las ventajas existentes en una explicación en gran grupo, concienciar de que esta metodología y otras muchas llevan muchos años inventadas y que serán ellos los que en el día de mañana deban utilizarlas en las aulas fomentando las competencias básicas de sus alumnos y alumnas y la importancia que tiene para los futuros docentes perder el miedo escénico a la hora de transmitir al resto de compañeros sus investigaciones, opiniones, trabajos monográficos, etc. facilitando de este modo la construcción social del conocimiento, adquiriendo de forma inconsciente una competencia fundamental en nuestra profesión: la competencia comunicativa. El papel del docente resulta fundamental en el desarrollo de esta importantísima competencia profesional en los estudiantes de magisterio, teniendo que cambiar la postura de profesor tradicional. "Si quieres que te escuchen di cosas interesantes para el grupo. Hazlos participar de forma activa en proyectos y decisiones". (Vidal y Fuertes, 2013: 8-9).

Con ello se consiguió familiarizarlos de modo general con la metodología a seguir durante el desarrollo de las dos asignaturas sin que supieran en ningún momento que las citadas metodologías serían objeto de análisis al final del cuatrimestre y donde los resultados arrojados por la investigación se les darían a conocer una vez terminada la docencia y su participación en las mismas. A parte de las sesiones magistrales y para evitar la anarquía pedagógica, se les facilitó posteriormente una guía de aprendizaje específica diseñada para tal fin donde se explicaban los principales objetivos de la metodología individualizada y el aprendizaje cooperativo así como las actividades a realizar, pautas de presentación, normas de citas bibliográficas, etc. siempre resaltando la importancia de su autonomía a la hora de realizarlas y partir de temas que les resultaran motivantes y relevantes para su formación como futuros docentes, insistiendo en la importancia de su implicación en el proceso de enseñanza-aprendizaje como garantía de éxito en éstas y otras asignaturas.

La tarea principal que se diseñó para implicar al alumnado como corresponsables con participación activa en el proceso de enseñanza-aprendizaje y evaluación fue la realización de un portafolios digital individual (blog) donde pudieran ir haciendo un seguimiento de las asignaturas, siendo consciente que "los nuevos soportes de 
comunicación en formato escrito, utilizados especialmente entre los jóvenes, como -bitácoras o blogs-" (Oliva, 2012: 72) o los dispositivos móviles son herramientas de uso diario para la mayoría de nuestros estudiantes utilizadas para introducir entradas significativas para ellos, compartir sus direcciones electrónicas con el resto de compañeros y compañeras, comentar noticias, escribir artículos, publicar sus presentaciones power point, etc. pero dando total libertad al diseño, organización, número de entradas y publicaciones, número de horas semanales dedicadas al mismo... De esta forma se pretendía ofrecer libertad al alumnado pero siempre sabiendo que era una actividad evaluable y lógicamente comparable con los realizados por el resto de compañeros, dando lugar una evaluación rápida y fácil al ser compartida de forma grupal. Como toda actividad novedosa, supuso un reto tanto para el profesor como para el alumnado menos habituados a manejar las TICE como herramienta de trabajo individual. Resulta imprescindible en esta parte conocer las palabras de Vigostky acerca de las zonas de desarrollo próximo y su importancia en la construcción del conocimiento.

La distancia entre el nivel actual de desarrollo, determinado por la capacidad de resolver independientemente un problema, y el nivel de desarrollo potencial, determinado a través de la resolución de un problema con la orientación de un adulto o la colaboración de un compañero más capaz. 1

Las tutorías individualizadas, la mediación del profesor y, en algunos casos, el trabajo cooperativo con alumnos más capaces permitió en un breve espacio de tiempo que toda la clase manejase su portafolios digital al menos de manera básica, ya que el objetivo principal de la tarea no era el manejo profesional de la herramienta tecnológica sino el uso adecuado de la misma para la adquisición de conocimientos, el desarrollo de competencias, la creación de comunidades de aprendizaje, el desarrollo de su juicio crítico, la búsqueda de información pertinente para el buen desarrollo de la asignatura y principalmente fomentar la competencia de aprender a aprender que en palabras de Delors "la educación está llamada a desarrollarse a lo largo de toda la vida” (Delors, 1996: 100).

Resulta interesante comprobar como desde hace años se viene desarrollando esta teoría sin que desde nuestro sistema educativo ni alumnado ni profesorado hayamos puesto demasiado empeño en poner en práctica formas de aprender y enseñar diferentes a las habitualmente conocidas. La individualización del aprendizaje es una de las metodologías que más han dado que hablar en ámbitos educativos desde hace más de 20 años con la aparición de la Ley Orgánica 1/1990, de 3 de octubre, de Ordenación General del Sistema Educativo, más conocida como LOGSE. Esta ley ya defendía en sus principales planteamientos un cambio en las metodologías docentes y en los roles educativos, haciendo al alumnado más protagonista de su proceso de enseñanza-aprendizaje y al profesor/a un guía; mediador entre lo que los alumnos ya conocen y lo que pueden aprender a través de su intervención educativa entendida como ayuda en esa construcción propia y social del conocimiento.

Respecto a los trabajos en grupo cooperativo, se establecieron tutorías presenciales de dos horas de duración, en las que el grupo tenía que desarrollar las tareas indicadas 
en la guía de trabajo mientras el tutor observaba y evaluaba el desarrollo de la sesión y el cumplimiento de los objetivos planteados, mediando e interviniendo pero no imponiendo las temáticas a tratar.

Unos días antes de la última tutoría había que colgar en el foro el trabajo esbozado, con las citas bibliográficas referenciadas en el formato requerido (normas APA) y se preparaba la presentación con la libertad de elegir cualquier tipo de formato, orientados por el profesor al uso de las TIC's por su carácter motivador e innovador pero, al igual que con la tarea individual, teniendo siempre presente las necesidades y capacidades de cada grupo así como sus intereses y motivaciones. De esta forma de trabajo, con el uso de esta metodología, podemos conseguir un doble aunque ambicioso objetivo, que los futuros docentes sean un motor de cambio metodológico y didáctico en las aulas del futuro "cuyas consecuencias derivadas de dicha intervención en la práctica educativa, tanto para el profesorado como para el alumnado" (Rodríguez Torres, 2013: 8) puedan convivir con otras perspectivas más tradicionalistas como la cultura del libro de texto editorial, pero que poco a poco la formación inicial del profesorado provoque cambios metodológicos en favor del aprendizaje globalizado y potencie la adquisición de competencias básicas y transversales y el uso de las TICE ya que "como hemos comprobado el mundo web cada vez hace más patente su “imprescindibilidad" en el mundo académico" (Martínez, 2012: 22).

\section{Resultados}

La exposición de los resultados se va a realizar separando los datos por asignaturas con el objetivo de poder demostrar como la misma metodología puede ser eficaz en materias diferentes, con características propias y contenidos lógicamente diferentes. En primer lugar se analiza "Tendencias Contemporáneas de Educación", asignatura de corte teórico donde la implicación del alumnado se hace fundamental para evitar la desmotivación propia de abusar de clases magistrales y del estudio de apuntes escritos. Tras el vaciado de los datos proporcionados por los estudiantes (25 mujeres y 17 varones), se han separado los resultados en dos bloques, por un lado el interés y satisfacción con la metodología empleada y por otro su opinión respecto a la misma, terminando con una valoración numérica global de la metodología, que nos proporcionará una autoevaluación para futuras mejoras docentes. 
Gráfico 1. Interés y satisfacción con la metodología empleada.



Elaboración propia

Como se puede comprobar (gráfico 1), el interés y satisfacción del alumnado con la metodología empleada arroja datos bastante satisfactorios en un porcentaje muy elevado. En estos seis primeros ítems que se muestran, el 40,47\% optaron por la opción "mucho" del cuestionario empleado (tabla 1) y el 52,38 se decantaron por la opción "bastante". Por el contrario, únicamente el 7,14\% señalaron la opción "poco" y ningún alumno o alumna optó por la opción "nada". Cabe señalar como especialmente significativos los datos arrojados en los ítems 1,2 y 5 , demostrando el elevado interés por la actividad individual propuesta (favoreciendo la implicación del alumnado en su proceso de enseñanza-aprendizaje), la satisfacción con el conocimiento adquirido en una materia de corte teórico y a priori poco motivante y la satisfacción por elegir libremente los temas a trabajar de forma grupal, partiendo de sus propias motivaciones, capacidades y conocimientos previos. 
Gráfico 2. Opinión respecto a la metodología

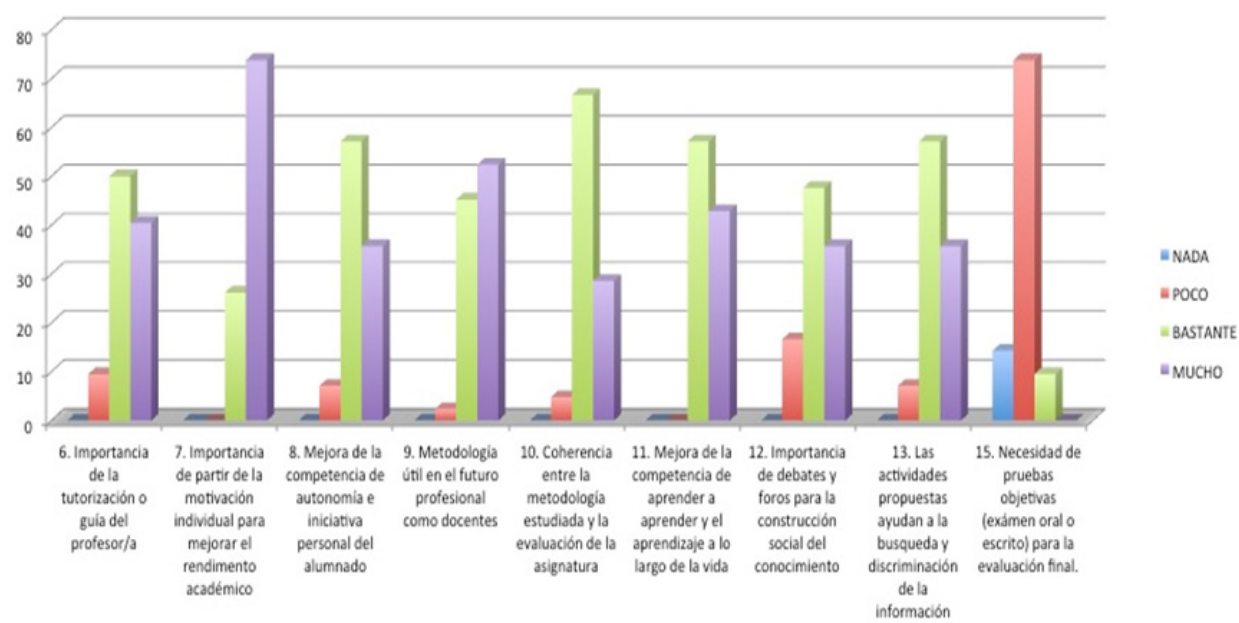

Elaboración propia

Para el análisis de este segundo gráfico, se va a comenzar por el ítem 15. La razón fundamental es la significatividad del resultado obtenido en el vaciado de datos. Como puede verse (gráfico 2), un elevadísimo porcentaje del alumnado señaló como poco o nada necesaria la utilización de pruebas objetivas convencionales (exámenes) dentro de la metodología de la individualización del aprendizaje y el trabajo cooperativo, pudiendo extraer la conclusión que la evaluación del proceso de enseñanza-aprendizaje puede y debe realizarse de formas alternativas a las tradicionales.

Una vez analizado este ítem, tan significativo en mi opinión, se analiza de forma global el resto de los ítems que evalúan la opinión respecto a la metodología y su repercusión en la mejora de las competencias consideradas importantes para el desempeño profesional.

El 43,11\% del alumnado se mostró muy satisfecho con la metodología y el 50,9\% bastante satisfecho. Datos muy esperanzadores para continuar con futuros estudios y mejoras al mismo.

Únicamente el 5,95\% de los estudiantes opinaron que la metodología era poco adecuada y nadie señaló la opción "nada" en ninguno de los ítem analizados. 
Gráfico 3. Resumen de datos asignatura Tendencias Contemporáneas de Educación.

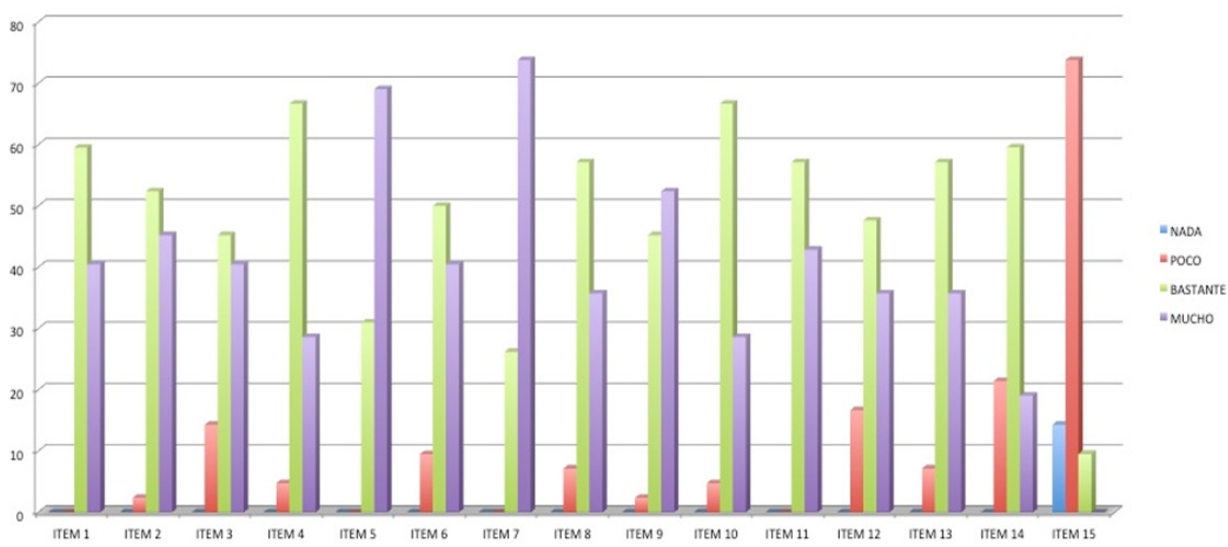

Elaboración propia

La metodología en esta asignatura fue valorada con un 8,375 de media por los por 42 alumnos/as que realizaron el cuestionario.

Para concluir con esta primera parte del análisis de resultados, se introduce un ejemplo de un blog (actividad individualizada propuesta) realizado por uno de los alumnos con la guía o mediación del profesor en la asignatura de corte más teórico de las dos estudiadas en la presente investigación: http://jaimejimenez1.wix.com/ nuestrastendecias

La segunda asignatura a analizar es la asignatura "Educación y Sociedad" (gráfi$\cos 4,5$ y 6). Esta asignatura, a diferencia de la anterior, tiene un perfil más práctico y motivante para el alumnado al combinar actividades más tradicionales con otras mucho más actuales. Como ya se ha comentado, la elaboración de portafolios digitales (blogs) por parte del alumnado fue, como en la anterior asignatura citada, la principal tarea individual a realizar por los alumnos. En ésta, al igual que en la anterior, se ha utilizado la misma metodología con variaciones en algunas actividades o tareas, pero bajo los mismos principios pedagógicos de partir de la motivación del alumnado, tutorización o mediación en los trabajos grupales, buscando un aprendizaje significativo más allá de la mera superación de la materia.

Tras el vaciado de los datos proporcionados por los estudiantes (25 mujeres y 18 varones), se han separado los resultados en dos bloques, por un lado el interés y satisfacción con la metodología empleada y por otro su opinión respecto a la misma, terminando con una valoración numérica global. 
Gráfico 4. Interés y satisfacción con la metodología empleada.

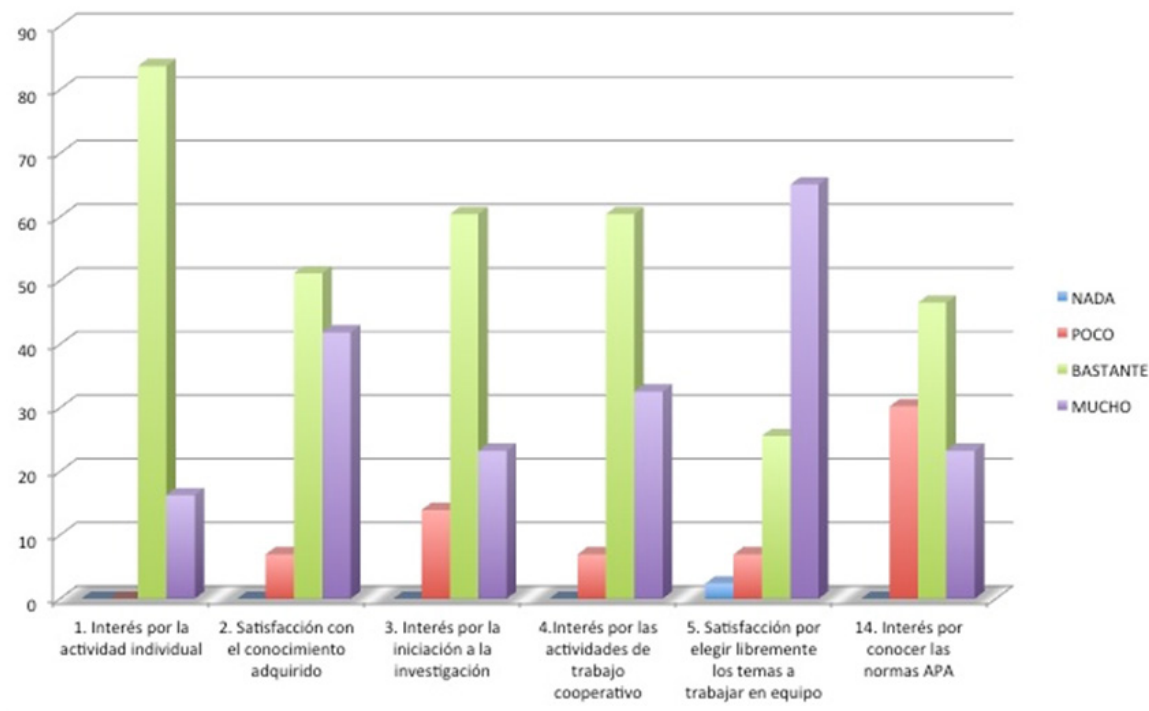

Elaboración propia

El 33,72\% del alumnado mostró mucho interés y satisfacción en términos generales con la propuesta metodológica en esta asignatura. Los estudiantes en un 54,65\%, se decantaron por la opción de "bastante" (tabla 1). Con estos datos se puede afirmar que el interés y la satisfacción del alumnado con la metodología estudiada es bastante o muy elevado (gráfico 4).

Por el contrario, solamente el $10,85 \%$ señalaron mostrarse poco satisfechos y únicamente el $0,39 \%$ mostró nulo interés y ninguna satisfacción con la metodología.

Respecto a la opinión del alumnado, el 40,11\% muestra una muy favorable valoración de la misma. También mostraron una opinión bastante favorable respecto a la metodología el 50,29\%. Estos datos no dan lugar a dudas puesto que representan un altísimo porcentaje del alumnado participante y que contestó al cuestionario. Llaman la atención principalmente la importancia de partir de la motivación individual para mejorar el rendimiento representado en el ítem 7 (gráfico 5) y la mejora de las competencias evaluadas en los ítem 8, 9 y 11.

El 7,26\% mantiene una opinión poco positiva de la metodología y únicamente el $0,29 \%$ optó por valorar ésta como nada interesante en su formación como futuros docentes. 
Gráfico 5. Opinión respecto a la metodología.

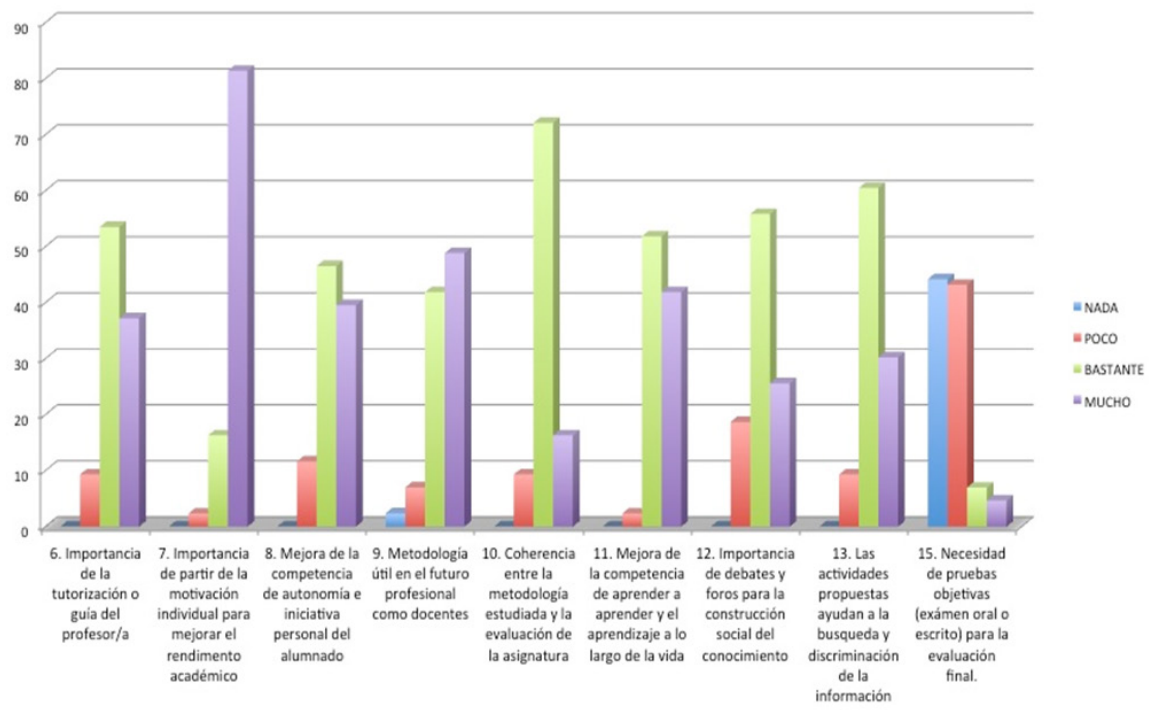

Elaboración propia

El análisis de este segundo gráfico y como se hizo anteriormente (gráfico 2) de la asignatura "Tendencias Contemporáneas de Educación" se va a concluir con el ítem 15 (gráfico 5). En este caso también es muy significativo el elevado porcentaje de alumnos y alumnas que señaló como poco o nada necesaria la utilización de pruebas objetivas convencionales dentro de la metodología de la individualización del aprendizaje y el trabajo cooperativo. Parece comprobarse que este tipo de metodología docente implica cambios en los roles tanto de docentes como de discentes, pero también en partes tan significativas del currículum como la programación de las actividades, los agrupamientos y la evaluación, tanto del alumnado como de la propia actuación docente.

La metodología en esta asignatura fue valorada con un 8,176 de media por los por 43 alumnos/as que realizaron el cuestionario. Para ilustrar la actividad individual propuesta en esta materia, se introduce un ejemplo que, al igual que en la anterior asignatura, se llevó a cabo bajo los principios de la individualización, motivación e interés de la alumna y la mediación o guía del profesor: http://beagarciaperez.blogspot.com.es/ 
Gráfico 6. Resumen de datos asignatura Educación y Sociedad.

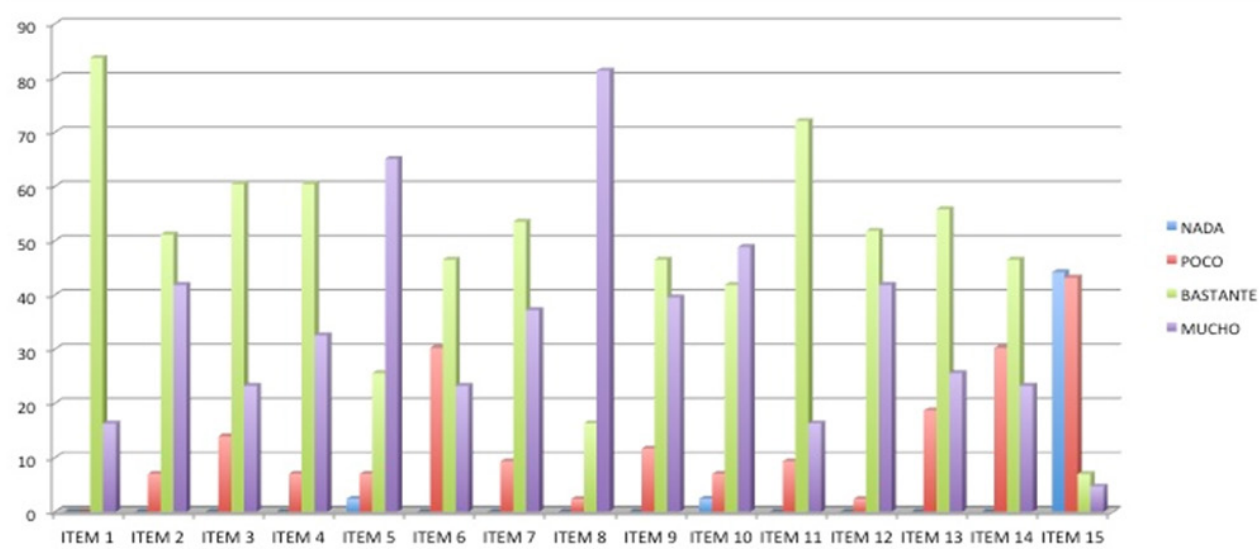

Elaboración propia

\section{Conclusiones}

La importancia que tiene para el proceso de enseñanza-aprendizaje la motivación del alumnado y, por qué no decirlo, la del docente parece fuera de toda duda. Los docentes que ya hayan trabajado con esta metodología habrán podido comprobar los beneficios que reporta la utilización de la misma en diferentes niveles educativos que, aunque no totalmente idénticos por la diferencia de edades e inquietudes de los educandos, sabrán como la individualización del aprendizaje unido al aprendizaje cooperativo es hoy en día una de las maneras más eficaces -y quizá también la más justa- de trabajar en las aulas diversas y multiculturales del siglo XXI.

Siguiendo con esta línea y comparándolo con investigaciones similares, se puede afirmar que tanto las competencias transversales como las propias del futuro profesional en la enseñanza son bien valoradas por los alumnos, competencias tan importantes como la de aprender a aprender, la autonomía e iniciativa personal, el aprendizaje a lo largo de la vida o la iniciación a la investigación. Al ser los resultados similares en ambas asignaturas e ir en la misma línea de estudios similares, podemos inferir que la metodología estudiada puede influir positivamente en la satisfacción con el conocimiento adquirido por parte de los estudiantes así como la importancia de partir de la motivación individual para mejorar el tan denostado rendimiento académico, algo que, sin ser por supuesto definitivo, sí da ciertas pautas para continuar en el trabajo al haber realizado la investigación en dos grupos distintos y en dos asignaturas diferentes con sus características intrínsecas propias, contenidos concretos y criterios de evaluación específicos para cada una de ellas obteniendo resultados similares, pero siempre bajo la misma metodología de trabajo y rompiendo, en cierta medida, con las tendencias más tradicionales de la educación. 


\section{Referencias bibliográficas}

DELORS, J. (1996). Informe Delors. La educación encierra un tesoro. Madrid: Unesco-Santillana.

DOLLASE, R.H. (1996). "The Vermont experiment in state-mandated portfolio program approval". En: Journal of Teacher Education, $n^{\circ}$ 47. p. 85-100.

DURAN MEDINA, J. F. (2012). Los Edublog en las aulas. Motivación asegurada (Vol. I). Madrid: Editorial Académica Española.

FREIRE, P. (1998). Pedagogía de la autonomía. Madrid: Siglo XXI Editores.

KLENOWSKI, V. (2000). "Portfolios: Promoting teaching. Assessment in Education". En: Principles, Policies, and Practice, $\mathrm{n}^{\circ}$ 7. p. 215-236.

MARTÍNEZ, S. (2012). "Periódicos universitarios en internet: los diarios de alumnos como herramienta docente en el nuevo entorno tecnológico". En: Revista de Comunicación de la SEECI, $\mathrm{n}^{\circ}$ 29. Madrid: UCM. p. 17-30.

MOKHTARI, D. y YELLIN, D. (1996). "Portfolio assessment in teacher education: Impact on preservice teachers' knowledge and attitudes". En: Journal of Teacher Education, n $^{\circ}$ 47. p. 245-248.

OLIVA, C. (2012). "El lenguaje como referente de la traslación del discurso oral al entorno digital: un espacio multimedia e interactivo". En: Revista de Comunicación Vivat Academia, $\mathrm{n}^{\circ} 120$. Madrid: UCM. p. 65-79.

RIVIÈRE, Á. (1984). La psicología de Vigotsky: sobre la larga proyección de una corta biografia. Madrid: Infancia y aprendizaje. p. 27-28.

RODRÍGUEZ, J. (2013). "Encrucijada docente: la inclusión de las tecnologías de la información y comunicación". En: Revista de Comunicación de la SEECI, n 31 . Madrid: UCM. p. 1-13.

VIDAL, S. y FUERTES, Ma. T. (2013). "La dinámica de grupos para el trabajo cooperativo facilita la comunicación”. En: Revista de Comunicación Vivat Académica, $\mathrm{n}^{\circ} 123$. P. $1-12$.

VISO, J. R. (2010). Enseñar y aprender por competencias. Vol. I. ¿Qué son las competencias?. Madrid: Editorial EOS.

\section{Notas}

${ }^{1}$ RIVIÈRE, Á. (1984). La psicología de Vigotsky: sobre la larga proyección de una corta biografía. Madrid: Infancia y aprendizaje. p. 27-28.

Se introduce esta nota citada por Ángel Rivière como sugerencia de que ciertas innovaciones pedagógicas como la tutoría entre iguales y el trabajo cooperativo fueron introducidas por autores como Vigostky en la llamada corriente pedagógica del constructivismo hace muchos años, perdurando hoy día otras corrientes o metodologías docentes más memorísticas o de transmisión directa de conocimientos. 


\section{El autor}

Álvaro Martín Espinosa es Diplomado en Magisterio por la UCLM con habilitaciones en las especialidades de primaria, educación física y pedagogía terapéutica. Licenciado en Psicopedagogía por la U.N.E.D. y Máster en Intervención Educativa en Contextos Sociales. A nivel profesional he desarrollado mi actividad docente no universitaria en distintos centros educativos desde el año 2002 y desde el 2009 soy profesor asociado en la UCLM, perteneciente al Departamento de Pedagogía, impartiendo docencia en asignaturas del Área de DOE y de Teoría e Historia de la Educación. He participado en diversos grupos de investigación relacionados principalmente con las TICE así como en varias publicaciones especializadas. 


\title{
Educación Mediática e Informacional en el contexto de la actual Sociedad del Conocimiento
}

\author{
Clara JANNETH SANTOS MARTíNEZ \\ Universidad Autónoma de Bucaramanga - UNAB \\ csantos110@unab.edu.co
}

\begin{abstract}
Resumen
La Educación Mediática e Informacional se reclama como núcleo central de las competencias clave para el Aprendizaje Permanente, para afrontar el siglo XXI en la actual Sociedad del Conocimiento. La importancia que ha cobrado este concepto expuesto en foros internacionales, especialmente durante los últimos 30 años, permite entender la forma como se asumen las TIC desde lo educativo -superando lo tecnológico-, y la necesidad de incluirlo como elemento transformador con un papel esencial en la educación, el aprendizaje permanente, la democracia y los derechos humanos. Este artículo invita a profundizar en su génesis y conformación para una mayor apropiación conceptual.
\end{abstract}

Palabras clave: Educación mediática; Alfabetización Mediática e Informacional; AMI; Alfabetizaciones múltiples; Sociedad del Conocimiento

\section{Media and Information Literacy inside the Society of Knowledge's context}

\begin{abstract}
Education Media and Information is claimed as the core of key competences for Lifelong Learning which will allow meeting the current century in the Knowledge Society. In the last 30 years this concept has gained worldwide importance and it allows us understand how ICT are assumed from the educational, overcoming the technological-, and the need to include it as a transformer with an essential role in Education, Lifelong Learning, Democracy and Human Rights. This article seeks to explore the genesis and shaping for greater conceptual appropriation
\end{abstract}

Key words: Media and Information Literacy; MIL; Multiple Literacies; Society of Knowledge

\section{Referencia normalizada:}

Santos Martínez, C. J. (2013) Educación Mediática e Informacional en el contexto de la actual Sociedad de la Información. Historia y Comunicación Social. Vol. 18. № Especial Diciembre. Págs. 781-795.-

Sumario: 1. Introducción; 2. Metodología; 3. El contexto de la globalización; 4. El contexto de la educación mediática; 5 . Conclusiones

\section{Introducción}

Aunque la importancia de las Tecnologías de la Información y la Comunicación, TIC, se vea respaldada por sus niveles de penetración mundial (2.500 millones de 